\title{
A Novel cDNA-uPA/SCID/Rag2-/-/Jak3/-- Mouse Model for Hepatitis Virus Infection and Reconstruction of Human Immune System
}

\section{Takuro Uchida}

Hiroshima University

\section{Yuji Teraoka}

Hiroshima University

\section{Michio Imamura}

Hiroshima University

\section{Hatsue Fujino}

Hiroshima University

\section{Atsushi Ono}

Hiroshima University

Takashi Nakahara

Hiroshima University

Eisuke Murakami

Hiroshima University

Masami Yamauchi

Hiroshima University

Tomokazu Kawaoka

Hiroshima University

Daiki Miki

Hiroshima University

Hiromi Abe-Chayama

Hiroshima University

Grace Naswa Makokha

Hiroshima University

C. Nelson Hayes

Hiroshima University

Hiroshi Aikata

Hiroshima University

\section{Satoko Hamamura}

PhoenixBio Co., Ltd 


\section{Yuji Ishida}

PhoenixBio Co., Ltd

\section{Chise Tateno}

PhoenixBio Co., Ltd

\section{Takayuki Shirouzu}

Wakunaga Pharmaceutical Co., Ltd

\section{Shintaro Kawai}

Wakunaga Pharmaceutical Co., Ltd

\section{Yuka Tanaka}

Hiroshima University

\section{Hideki Ohdan}

Hiroshima University

\section{Seiji Okada}

Kumamoto University

Kazuaki Chayama ( $\nabla$ chayama@hiroshima-u.ac.jp)

Hiroshima University

\section{Research Article}

Keywords: humanized mouse, pathogenesis, drug development, hepatocytes, human leukocyte antigen (HLA)

Posted Date: July 20th, 2021

DOl: https://doi.org/10.21203/rs.3.rs-712014/v1

License: (c) (1) This work is licensed under a Creative Commons Attribution 4.0 International License. Read Full License 


\section{Abstract}

The humanized mouse is a widely used in vivo model for the investigation of pathogenesis and drug development. In this study, we generated new immunodeficiency cDNA-urokinase-type plasminogen activator (UPA)/SCID/Rag ${ }^{-/} / \mathrm{Jak}^{-/-}$mice and established the mouse model with a humanized liver and immune system. Transplantation of human hepatocytes with human leukocyte antigen (HLA)-A24 resulted in establishment of a highly replaced liver in this mouse model. These mice were successfully infected with hepatitis $B$ virus and hepatis $C$ virus $(\mathrm{HCV})$ for a prolonged period and are available for the analysis of the effect of anti-HCV drugs. Administration of peripheral blood mononuclear cells (PBMCs) obtained from a donor with HLA-A24 resulted in the establishment of 22.6-81.3\% of human CD45positive mononuclear cell chimerism in liver infiltrating cells without causing graft versus host disease. When mice were transplanted with human hepatocytes and then administered PBMCs, an alloimmune response between transplanted human hepatocytes and PBMCs occurred, with production of transplanted hepatocyte-specific anti-HLA antibody. The alloimmunity was never inhibited by methylprednisolone nor cyclosporine A. In conclusion, we succeeded in establishing a humanized liver and immune system using a novel cDNA-uPA/SCID/Rag2 $2^{-/} / \mathrm{Jak3}^{-/-}$mouse. This model is not only useful to study hepatitis virus virology but also to study alloimmunity.

\section{Introduction}

The humanized mouse model is widely used for the exploration of disease pathogenesis and the evaluation of the therapeutic effect of drugs. Several immunodeficient mice have been developed in order to accept transplanted human cells. NOD/SCID/IL2 $\mathrm{Yc}^{-/-}$, such as NOG and NSG mice, are considered a suitable platform for in vivo reconstruction of the human immune system. However, it is known that the genetic background of these mice is influenced by the efficiency of human cell engraftment ${ }^{1}$. Rag2/Jak3 double-deficient ( $\mathrm{Rag}^{-/-} / \mathrm{Jak3}^{-/-}$) mice lacking mature $\mathrm{T}$ and $\mathrm{B}$ lymphocytes and NK cells, is a suitable mouse model for reconstruction of human immune system by transplantation of human immune cells ${ }^{2}$.

Hepatitis B virus (HBV) and hepatitis C virus (HCV) pose a worldwide health burden causing acute hepatitis, chronic hepatitis, liver cirrhosis, and potentially death due to liver failure and hepatocellular carcinoma. The species tropism of these viruses is restricted to chimpanzee and human; thus, investigation of their pathology had been hampered by the lack of small animal model. Recently developed human hepatocyte replaced immunodeficiency mice, such as urokinase-type plasminogen activator (UPA) ${ }^{2,3}$, herpes simplex virus type- 1 thymidine kinase (HSVtk) ${ }^{4}$ transgene, or a targeted disruption of the murine fumaryl acetoacetate hydrolase (FAH) ${ }^{5,6}$ support hepatitis viruses infection and replication and are commonly used for the study of viral hepatitis ${ }^{7-9}$. We previously reported that TKNOG and CDNA-UPA/SCID mice were useful animal models for the study of HBV and HCV virology and the analysis of the effect of antiviral drugs ${ }^{10,11}$. Moreover, we established the hepatitis $B$ mouse model by the adoptive transfer of human immune cells to human hepatocyte replaced mice ${ }^{12,13}$. 
In this study, we generated novel CDNA-uPA/SCID/Rag2 $2^{-/-} / \mathrm{Jak}^{-/-}$mice with humanized livers that were almost completely repopulated with human hepatocytes, and we are able to successfully infect these mice with HBV and HCV. In addition, we attempted to reconstruct elements of the human immune system in this novel mouse model by administration of human peripheral blood mononuclear cells (PBMCs).

\section{Results}

\section{Characteristics of CDNA-uPA/SCID/Rag2 $2^{-/-} / \mathrm{Jak3}^{-/-}$mice}

The pregnant ratio of $\mathrm{CDNA}-\mathrm{uPA} / \mathrm{SCID} / \mathrm{Rag}^{-/-} / \mathrm{Jak}^{-/-}$mice was very low when the Jak3 knockout was

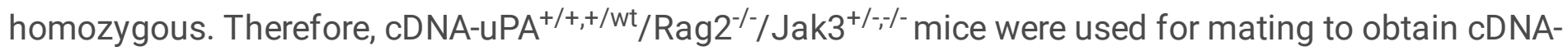
uPA/SCID/Rag2 ${ }^{-/} / \mathrm{Jak}^{-/-}$mice. The male : female sex ratios of cDNA-uPA/SCID/Rag2 $2^{-/-} / \mathrm{Jak3}^{-/-}$mice were $4.1: 5.9$ and $3.5: 6.5$ for cDNA-uPA homozygous $(n=99)$ and hemizygous $(n=120)$, respectively. These ratios were similar to those for CDNA-UPA/SCID mice $(4: 6)$.

\section{Repopulation of CDNA-uPA/SCID/Rag2 $2^{-/-} / \mathrm{Jak}^{-/-}$mice with humanized livers}

When the mice were 2-4 weeks of age, $5.0 \times 10^{5}$ viable human hepatocytes were isolated from human hepatocyte-replaced cDNA-uPA/SCID mice (PXB-mice $\left.{ }^{\circledR}\right)$ by collagenase perfusion method and

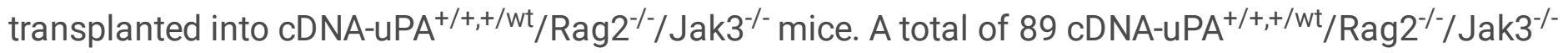
mice were transplanted with human hepatocytes. Changes in the body weight and serum HSA levels of 13-week-old mice are shown in Figure 1 and Table 1. The HSA levels increased steadily after transplantation of human hepatocytes and reached a steady state at around 11 weeks (Fig. 1A). Mice serum HSA levels were higher in CDNA-uPA homozygote chimeric mice compared to hemizygote chimeric mice from 6 weeks old ( 3 weeks after transplantation) to 13 weeks old, indicating that engraftment of human hepatocytes was superior in cDNA-uPA homozygote host mice. While body weights were higher in cDNA-uPA hemizygote mice than homozygote mice (Fig. 1B). Mean HSA levels in males were slightly higher than those in females of both cDNA-uPA homozygote and hemizygote human hepatocyte-replaced mice (Table 1). The correlation between HSA levels at 17 weeks old and the human hepatocyte replacement index, estimated by immunohistochemistry in cDNA-uPA homozygote mice, is shown in Figure 1B. HSA levels were exponentially correlated with replacement index in cDNAuPA/SCID/Rag2 $2^{-/-} / \mathrm{Jak3}^{-/-}$mice $\left(y=35.0 \mathrm{ln}(\mathrm{x})-475.0, \mathrm{r}^{2}=0.92\right)$ in a manner comparable to PXBmice ${ }^{\circledR}\left(y=29.9 \ln (x)-397.0, r^{2}=0.88\right)^{2}$. The formula indicated that a level of more than 6.2 $\mathrm{mg} / \mathrm{mL}$ HSA represents a replacement index exceeding $70 \%$ in human hepatocyte transplanted cDNAuPA/SCID $/$ Rag $^{-/} /$Jak3 $^{-/-}$mice.

\section{Hepatitis virus infection to human hepatocyte-transplanted cDNA-uPA/SCID/Rag2 ${ }^{-/-} / \mathrm{Jak}^{-/-}$mice}

Each of six mice was inoculated with $1 \mu \mathrm{L}$ of serum samples containing $10^{6}$ copies of HBV. Mice serum HBV DNA became positive two weeks after HBV inoculation, increased gradually, and reached $10^{8}-10^{10}$ copies/mL at 6-12 weeks (Fig. 2A). High levels of HBV DNA and HSA persisted until 30-37 weeks. 
Each of four human hepatocyte-replaced cDNA-uPA/SCID/Rag2 $2^{-/} / \mathrm{Jak}^{-/-}$mice was injected with $10 \mu \mathrm{L}$ of serum samples containing $10^{5}$ copies of HCV in order to establish HCV viremia. Mice serum HCV RNA became measurable two weeks after HCV inoculation and reached $10^{6}-10^{8}$ copies $/ \mathrm{mL} 10$ weeks after $\mathrm{HCV}$ inoculation (Fig. 2B). When these mice were administered either the NS5A inhibitor daclatasvir or the NS3/4A protease inhibitor asunaprevir, viral breakthrough developed following a transient reduction in HCV RNA levels. These results indicate that HCV-infected CDNA-uPA/SCID/Rag2 $2^{-/} / \mathrm{Jak}^{-/-}$mice are useful for evaluating antiviral agents in vivo in a similar way to previous human hepatocyte chimeric mouse models.

\section{Reconstruction of the human immune system in CDNA-uPASCID/Rag2 $2^{-1 /} / \mathrm{Jak}^{-/-}$mice}

Human hepatocyte-transplanted CDNA-uPA/SCID/Rag2 $2^{-/-} / \mathrm{Jak}^{-/-}$mice tolerate infection with hepatitis viruses and seem to be suitable animal models for evaluating the effect of antiviral drugs. However, the models are less likely to develop viral hepatitis due to the lack of an immune system. Human PBMCs were transplanted into CDNA-UPA/SCID/Rag2 $2^{-/ /} / \mathrm{Jak}^{-/-}$mice in order to establish a basic immune system. Characteristics of human PBMCs used in this study are shown in Table 2. First, $5 \times 10^{6}$ PBMCs obtained from a healthy volunteer (Case 1 ) were administered into three cDNA-uPA/SCID/Rag2 $2^{-/ /} / \mathrm{Jak}^{-/-}$mice without human hepatocyte transplantation. Mouse body weight was maintained at baseline level after PBMC administration (Fig. 3A). Flow cytometry analysis revealed the establishment of $81.3 \%$ and $22.6 \%$ of human CD45-positive mononuclear cell chimerism in liver infiltrating cells 4 and 8 weeks after PBMC transplantation in two mice, respectively (Fig. 3B).

We next administered human PBMCs obtained from Case 1 (healthy volunteer) into four human hepatic hepatocyte-transplanted cDNA-uPA/SCID/Rag2 $2^{-/ /} / \mathrm{Jak}^{-/-}$mice. Mice developed rapid and severe weight loss, ruffled hair and reduced activity following PBMC transplantation (Fig. 4A). Flow cytometry analysis revealed an engraftment of $30.4 \%$ of human CD45-positive mononuclear cell in liver infiltrating cells in a mouse (Fig. 4B), and histological examination showed lymphocyte accumulation in livers, especially in human albumin-positive hepatocytes 19 weeks after PBMC transplantation (Fig. 4C). On the other hand, histological examination showed no evidence of development of graft versus host disease (GVHD) in multiple organs such as colon, small bowel, stomach, lung, kidney, pancreas, spleen and heart (Fig. 4D). Therefore, the symptoms following human PBMC administration seemed to be caused by alloimmunity between administered human PBMCs and transplanted human hepatocytes.

We also tested PBMC administration into HBV-infected CDNA-uPA/SCID/Rag2 $2^{-/ /} / \mathrm{Jak}^{-/-}$mice. Human hepatocyte-transplanted mice were infected with HBV and administered with PBMCs obtained from Case 1. Unfortunately, mice developed body weight loss, and mice serum HSA level decreased two weeks after PBMC administration similar to the experiment using uninfected mice. Although HBV DNA levels decreased in these mice in response to the reduction in HSA level, HBV-specific cytotoxic T lymphocytes (CTLs) were not detected in liver-infiltrating cells obtained from these mice (Fig. 5). These results indicate 
that reduction of human hepatocytes in HBV-infected CDNA-UPA/SCID $/ \mathrm{Rag}^{-1-} / \mathrm{Jak}^{-/-}$mice was mainly due to the alloimmunity between administered human PBMCs and transplanted human hepatocytes.

To search for a candidate whose PBMCs do not induce an alloimmune response with transplanted human hepatocytes, we tested PBMCs obtained from a patient who had recovered from an episode of acute severe hepatitis B (Case 2) and a patient with chronic HBV infection (Cases 3) (Table 2). Similar to the PBMCs obtained from Case 1, PBMC administration resulted in reductions in body weight, HSA and HBV DNA level in both cases (Fig. 5).

To inhibit alloimmunity, mice were treated with either or both methylprednisolone and cyclosporine A for 7 days after PBMC transplantation. However, both mice body weight and HSA levels decreased in spite of methylprednisolone, cyclosporine A, or combination of these drugs treatments (Fig. 6).

We measured HLA antibodies in the serum of cDNA-uPA/SCID/Rag2 $2^{-/-} / \mathrm{Jak3}^{-/-}$mice after PBMC administration to make sure that an alloimmune response occurred between administered human PBMCs and transplanted human hepatocytes. Anti-HLA-B61 antibody, which matched the HLA type of the transplanted human hepatocytes, was detected in three mice that were administered serum from Donors 1 and 2 (Table 3). On the other hand, this antibody was not detected in any of the cDNAuPA/SCID/Rag2 $2^{-/-} / \mathrm{Jak3}^{-/-}$mice that had not been transplanted with hepatocytes (data not shown). Although we detected non-specific anti-HLA antibodies in six out of ten mice that had been administered PBMCs, they were not detected in the remaining four mice. Non-specific anti-HLA antibodies detected in this study might be due to the presence of HLA-presenting cells in transplanted PBMCs. These results indicate that PBMC administration resulted in development of anti-HLA-B61 antibody and induced an alloimmune response to transplanted human hepatocytes in these mice.

\section{Discussion}

Human hepatocyte chimeric mice are widely used for research on HBV and HCV. Although this mouse model provides a platform for examining hepatitis virus infection, it is difficult to investigate the immune response to the viruses due to the lack of an immune system. In this study, we attempted to develop a novel chronic hepatitis $B$ mouse model using human hepatocytes transplanted into severe immunodeficiency, CDNA-uPA/SCID/Rag2 $2^{-/-} / \mathrm{Jak}^{-/-}$mice.

In human hepatocyte-transplanted cDNA-uPA/SCID/Rag2 $2^{-/-} / \mathrm{Jak}^{-/-}$mice, characteristics of HSA levels and body weight in homozygotes and hemizygotes and in males and females were similar to those of PXB mice ${ }^{\circledR}{ }^{2}$. HBV and HCV infection were supported in human hepatocyte-replaced CDNAUPA/SCID/Rag2 $2^{-/-} / \mathrm{Jak3}^{-/-}$mice with sustained high HSA levels, and serum HBV DNA and HCV RNA reached similar levels to those in PXB-mice ${ }^{\circledR}{ }^{11}$. Excellent repopulation of human hepatocytes resulted in high titers of viremia and allowed us to perform a long-term infection experiment in this mouse model. In addition, we administered daclatasvir and asunaprevir to HCV-infected human hepatocyte-replaced cDNA-uPA/SCID/Rag2 $2^{-/-} / \mathrm{Jak}^{-/-}$mice and confirmed the antiviral effects. These results suggest that 
this mouse model is useful for hepatitis virus infection experiments and the evaluation of antiviral agents in comparable way to PXB-mice ${ }^{\circledR}{ }^{11}$.

We next succeeded in engrafting human immune cells in cDNA-uPA/SCID/Rag2 $2^{-/-} / \mathrm{Jak3}^{-/-}$to reconstruct elements of a simple human immune system. Human immune cells were detected in mouse livers after PBMC administration, but mice developed the symptoms due to allo-response in human hepatocyte and immune cell dual chimeric mice with and without HBV infection. Although we tried to match the HLA haplotypes between human hepatocytes and PBMCs as much as possible, it was difficult to find candidates who have completely matched-HLA types. This phenomenon seemed to be mainly due to alloimmunity between transplanted human hepatocytes and administered human PBMCs because they were not observed in mice without human hepatocyte transplantation, and anti-HLA antibody was detected only in human hepatocyte transplanted mice. It is noteworthy that mismatch of HLA is one of the most important factors which led to the allo-reaction in this immune cell transplanted mouse model. Moreover, it is possible that an overreaction between human hepatocyte and transplanted PBMCs might be likely to occur due to the excellent repopulation of human hepatocytes.

For humanized hepatitis mouse models, it is reported that human hepatocyte and immune cells dual chimeric mouse model using hematopoietic stem cells obtained from cord blood could support HBV infection and investigation of human immune response to HBV-infected hepatocytes ${ }^{17}$. However, the human hepatocyte replacement ratio in this dual mouse model is considerably lower than in our chimeric mouse model. In addition, humanized mice with human induced pluripotent stem derived hepatic like cells are transplanted was reported ${ }^{18}$. Although stem cells also have the ability to differentiate into immune cells, the immune response model using stem cell derived immune cells is more complicated. Altogether, there are still many problems to overcome to establish a mouse model which mimic the viral hepatitis.

Meanwhile, the immune response between graft and host is a serious problem when constructing immune system in vivo models and is mainly thought to be caused by MHC class $1 / 2$ molecules ${ }^{19}$. Various xeno-immunity mouse models are reported using immunodeficiency mouse strains ${ }^{20}$. However, no alloimmunity mouse model was reported so far. According to the previous report, allo-specific anti-HLA antibody was difficult to detect in human immune system chimeric mouse model without humanized liver ${ }^{21}$. In our mouse model, we confirmed immune cell accumulation, especially in human hepatocyte areas and detection of transplanted human hepatocyte-specific anti-HLA antibody. Antibody-mediated allograft rejection due to donor-specific anti-HLA antibody influences transplantation outcomes despite advances in immunosuppression and antibody screening prior to the transplantation ${ }^{22,23}$. However, the mechanism of anti-HLA antibody-mediated rejection has not been fully elucidated so far. This mouse model might be helpful to investigate acute allo-reaction between transplanted human immune cells and human liver. Further study is needed to verify the mechanism of immune response in this mouse model. 
In conclusion, we established a novel cDNA-uPA/SCID/Rag2 $2^{-/-} / \mathrm{Jak}^{-/-}$mouse model which supports reconstruction of hepatitis virus infection and part of the human immune system. This mouse model is useful for investigation of the pathogenesis of chronic hepatitis virus infection and evaluation of the effects of antiviral compounds with the potential to mimic donor-specific anti-HLA antibody-mediated alloimmunity.

\section{Methods}

\section{Generation of $\mathrm{CDNA-uPA} S \mathrm{SID} / \mathrm{Rag2}^{-/-} / \mathrm{Jak3}^{-/-}$mice with humanized liver}

$\mathrm{Rag}^{-/ /} / \mathrm{Jak}^{-/-}$mice line with NOD, C57/BL6 and Balb/c genetic background were generated at Kumamoto University, Japan ${ }^{1}$. The generation of CDNA-UPA/SCID mice has been described previously ${ }^{2}$. CDNA-UPA/SCID mice were backcrossed twice with Rag2 $2^{-/ /} / \mathrm{Jak}^{-/-}$mice with Balb/c genetic background. Subsequently, they were mated together by caesarian operation to obtain microbiologically cleaned cDNA-uPA/SCID/Rag2 ${ }^{-/} / \mathrm{Jak}^{-/-}$mice. To prepare host mice for human hepatocyte replaced mice, cDNA-

$\mathrm{UPA}^{+/+}$or +/wild $/ \mathrm{SCID} / \mathrm{Rag}^{-/ /} / \mathrm{Jak}^{-/- \text {or }-/ \text { wild }}$ mice were mated with each other. The genotypes of uPA, Rag2 and Jak3 were analyzed by the genomic PCR method. CDNA-uPA ${ }^{+/+}$or +/wild $/$SCID $/$Rag $^{-/ /} / \mathrm{Jak}^{-/-}$mice were transplanted with human hepatocytes with human leukocyte antigen (HLA)-A24 (BD Biosciences, Woburn, MA, USA) at 2-4 weeks old. The thawing and transplantation of human hepatocytes into host mice has been described previously in the generation of chimeric mice with human hepatocytes ${ }^{4}$. All animal protocols described in this study were performed in accordance with the Guide for the Care and Use of Laboratory Animals (https://grants.nih.gov/grants/olaw/guide-for-the-care-and-use-of-laboratoryanimals.pdf) and the local committee for animal experiments, and the experimental protocol was approved by the Ethics Review Committee for Animal Experimentation of the Graduate School of Biomedical Sciences, Hiroshima University and PhoenixBio Co., Ltd. This study is reported in accordance with ARRIVE guidelines (https://arriveguidelines.org).

\section{Measurement of the replacement index and human serum albumin levels}

Cryosections prepared from the liver ( $5 \mu \mathrm{m}$ thick) were incubated with anti-human cytokeratin 8 and 18 (CK8/18) mouse monoclonal antibodies ('100 dilution, Cappel Laboratory, Cochranville, PA).

Measurements of the replacement index of human hepatocytes to total human and mouse hepatocytes have been described previously ${ }^{14}$. Mouse blood was collected from the tail vein. The concentration of human serum albumin (HSA) in mouse blood was measured using latex agglutination immunonephelometry (LX Reagent “Eiken” Alb Il; Eiken Chsemical Co., Ltd., Tokyo, Japan).

\section{HBV and HCV infections in mice}

Twelve weeks after human hepatocyte transplantation, cDNA-uPA/SCID/Rag2 $2^{-/ /} / \mathrm{Jak}^{-/-}$mice were injected intravenously with either HBV or HCV containing serum. HBV containing serum was obtained from an HBeAg-positive patient with chronic genotype C HBV infection who had high virus titers (8.2 log 
$\mathrm{IU} / \mathrm{mL})$. HCV containing serum was obtained from a patient with chronic genotype $1 \mathrm{~b}$ HCV infection with high virus titers (6.3 log IU/mL). After serum inoculation, mouse blood samples were obtained serially, and serum viral titers and HSA levels were measured. Human serum samples were obtained from patients who provided written informed consent using a form that was made in accordance with the Declaration of Helsinki and was approved by the ethical committee of Hiroshima University. The individual serum samples were divided into small aliquots and stored separately in liquid nitrogen until use.

\section{Quantification of HBV DNA and HCV RNA}

Serum HBV DNA and HCV RNA levels were measured using the COBAS TaqMan test (Roche Diagnostics). The lower detection limits of the assay for HBV DNA and HCV RNA are 4.4 and 3.5 log copies $/ \mathrm{mL}$, respectively.

\section{Treatment with direct-acting antivirals for HCV-infected mice}

After establishment of stable viremia, HCV-infected cDNA-uPA/SCID/Rag2 ${ }^{-/-} / \mathrm{Jak}^{-/-}$mice received orally either $40 \mathrm{mg} / \mathrm{kg} /$ day of asunaprevir (Bristol-Myers Squibb, New York, NY) or $10 \mathrm{mg} / \mathrm{kg} /$ day of daclatasvir (Bristol-Myers Squibb). The dose of each drug was the determined as the mouse equivalent dose used in our previous report (15?).

\section{Preparation of human PBMCs and transplantation into mice}

PBMCs were isolated using Ficoll-Paque density gradient centrifugation according to the manufacturer instructions. $5 \times 10^{6}$ of PBMCs were transplanted into CDNA-UPA/SCID/ Rag2 $2^{-/ /} / \mathrm{Jak}^{-/-}$mice by peritoneal injection.

\section{Flow cytometry}

We collected mouse liver-infiltrating cells flowing through the portal vein after hepatectomy ${ }^{16}$. Reconstructed human PBMCs in mice were analyzed by flow cytometry with monoclonal antibodies used for surface staining as described previously ${ }^{12,13}$. Dead cells identified by light scatter and 7-AAD staining were excluded from the analysis. Flow cytometry was performed using a FACS Aria II flow cytometer (BD Bioscience, Franklin Lakes, NJ), and results were analyzed with FlowJo (BD Biosciences).

\section{Histochemical analysis of mouse liver}

Histochemical analysis and immunohistochemical staining using antibodies against human albumin (Bethyl Laboratories Inc., Montgomery, TX) were performed as described previously ${ }^{12,13}$. Immunoreactive materials were visualized using a streptavidin-biotin staining kit (Histofine SABPO kit; Nichirei, Tokyo, Japan) and diaminobenzidine.

\section{Treatment of mice with immunosuppression}


One week after human PBMC transplantation, human hepatocyte-replaced cDNA-uPA/SCID/Rag2 ${ }^{-/} / \mathrm{Jak}^{-/-}$ mice received either or both of $50 \mathrm{mg} / \mathrm{kg} /$ day of cyclosporine A orally and intra-peritoneal injections of $200 \mathrm{mg} / \mathrm{kg}$ of methylprednisolone for seven days.

\section{Detection of anti-HLA antibody in mice}

Anti-human leukocyte antigen (HLA) antibody was detected using WAKFlow class $\$ antibody specificity identification reagent (Wakunaga Pharmaceutical Co., Ltd., ,Tokyo, Japan) according to the manufacturer's protocol. Data acquisition and analysis were performed by the Luminex® system (Luminex corporation, Austin, TX) to identify HLA alleles encoded by sample DNA.

\section{Abbreviations}

DAAs, direct acting antivirals; FAH, fumaryl acetoacetate hydrolase; GVHD, graft-versus-host disease; HBV, Hepatitis B virus; HCV, Hepatitis C virus; HLA, human leukocyte antigen; HSVtk, herpes simplex virus type1 thymidine kinase; PBMCs, peripheral blood mononuclear cells; UPA, urokinase-type plasminogen activator

\section{Declarations}

\section{Acknowledgments}

We thank Rie Akiyama and Yoko Inoue for their expert technical help; Emi Nishio for technical assistance. This research is partially supported by research funding from the Research Program on Hepatitis from the Japan Agency for Medical Research and Development, AMED (grant numbers: 20fk0310109h0004 and 20fk0210065h0001). Part of this work was carried out at the Analysis Center of Life Science, Natural Science Center for Basic Research and Development, Hiroshima University

\section{Author contribution}

Concept and design: TU, MI, and KC

Experiment, procedures and data analysis: TU, YT, MI, SH, YI and CT

Project administration: HF, AO, TN, EM, MY, TK, HAC, GNM, CNH, DM, AH, HA, YT, HO and KC

Provision of resources: $\mathrm{SH}, \mathrm{YI}, \mathrm{CT}$ and $\mathrm{SO}$

Measurement of anti-HLA antibody: TS and SK

Writing of manuscript: $\mathrm{TU}, \mathrm{YT}, \mathrm{Ml}, \mathrm{CNH}$, and $\mathrm{KC}$

Supervision and critical revision of manuscript: CT, HO, SO and KC 


\section{Funding statement}

This research is partially supported by research funding from the Research Program on Hepatitis from the Japan Agency for Medical Research and Development, AMED (grant numbers: 20fk0310109h0004 and $20 \mathrm{fk} 0210065 \mathrm{h0001}$ ). The funders had no role in study design, data collection and analysis, decision to publish, or preparation of the manuscript. There was no additional external funding received for this study.

\section{Conflict of interest statement}

Michio Imamura has received research funding from AbbVie. Hiroshi Aikata has received honoraria from Eisai and Bayer. Kazuaki Chayama has received honoraria from Bristol-Myers Squibb and MSD K.K., AbbVie, Gilead Science, Dainippon Sumitomo Pharma and Mitsubishi Tanabe Pharma and research funding from Gilead Science, Dainippon Sumitomo Pharma, MSD K.K., AbbVie, Eisai, TORAY, Otsuka Pharma, Chugai Pharma, Takeda Pharma and Roche. Satoko Hamamura, Yuji Ishida and Chise tateno are employees of PhoenixBio Co., Ltd. Takayuki Shioruzu and Shintaro Kawai are employee of Wakunaga Pharmaceutical Co., Ltd.. All the other authors have no COI to declare.

\section{References}

1. Ono, A. et al. Comparative study of human hematopoietic cell engraftment into Balb/c and C57BL/6 strain of rag-2/Jak3 double-deficient mice. J. Biomed. Biotechnol. 2011, 2-7 (2011).

2. Tateno, C. et al. Generation of Novel Chimeric Mice with Humanized Livers by Using Hemizygous cDNA-uPA/SCID Mice. PLoS One 10, e0142145 (2015).

3. Mercer, D. F. et al. Hepatitis C virus replication in mice with chimeric human livers. Nat. Med. 7, 92733 (2001).

4. Hasegawa, M. et al. The reconstituted 'humanized liver' in TK-NOG mice is mature and functional. Biochem. Biophys. Res. Commun. 405, 405-10 (2011).

5. Azuma, H. et al. Robust expansion of human hepatocytes in Fah-//Rag2-/-/I2rg-/- mice. Nat. Biotechnol. 25, 903-10 (2007).

6. Bissig, K.-D., Le, T. T., Woods, N.-B. \& Verma, I. M. Repopulation of adult and neonatal mice with human hepatocytes: a chimeric animal model. Proc. Natl. Acad. Sci. U. S. A. 104, 20507-11 (2007).

7. Allweiss, L. \& Dandri, M. Experimental in vitro and in vivo models for the study of human hepatitis $B$ virus infection. J. Hepatol. 64, S17-31 (2016).

8. Vercauteren, K., De Jong, Y. P. \& Meuleman, P. Animal models for the study of HCV. Curr. Opin. Virol. 13, 67-74 (2015).

9. Giersch, K. et al. Hepatitis Delta co-infection in humanized mice leads to pronounced induction of innate immune responses in comparison to HBV mono-infection. J. Hepatol. 63, 346-53 (2015).

10. Kosaka, K. et al. A novel TK-NOG based humanized mouse model for the study of HBV and HCV infections. Biochem. Biophys. Res. Commun. 441, 230-5 (2013). 
11. Okazaki, A. et al. Severe necroinflammatory reaction caused by natural killer cell-mediated Fas/Fas ligand interaction and dendritic cells in human hepatocyte chimeric mouse. Hepatology 56, 555-566 (2012).

12. Takenaka, K. et al. Polymorphism in Sirpa modulates engraftment of human hematopoietic stem cells. Nat. Immunol. 8, 1313-1323 (2007).

13. Uchida, T. et al. Usefulness of humanized cDNA-UPA / SCID mice for the study of hepatitis B virus and hepatitis C virus virology. J. Gen. Virol. 98, 1040-1047 (2017).

14. Kan, H. et al. Combination therapies with daclatasvir and asunaprevir on NS3-D168 mutated HCV in human hepatocyte chimeric mice. Antivir. Ther. 21, 307-15 (2016).

15. Schultze, J. L. et al. Follicular lymphomas can be induced to present alloantigen efficiently: a conceptual model to improve their tumor immunogenicity. Proc. Natl. Acad. Sci. U. S. A. 92, 8200-4 (1995).

16. Ohdan, H. et al. Intraoperative near-infrared spectroscopy for evaluating hepatic venous outflow in living-donor right lobe liver. Transplantation 76, 791-797 (2003).

17. Strick-Marchand, H. et al. A novel mouse model for stable engraftment of a human immune system and human hepatocytes. PLoS One 10, e0119820 (2015).

18. Dusséaux, M. et al. Viral Load Affects the Immune Response to HBV in Mice With Humanized Immune System and Liver. Gastroenterology 153, 1647-1661.e9 (2017).

19. King, M. A. et al. Human peripheral blood leucocyte non-obese diabetic-severe combined immunodeficiency interleukin-2 receptor gamma chain gene mouse model of xenogeneic graftversus -host-like disease and the role of host major histocompatibility complex. Clin. Exp. Immunol. 157, 104-118 (2009).

20. Huang, F., Cao, F. L. \& Zheng, S. G. Update of humanized animal disease models in studying Graftversus-host disease. Hum. Vaccines Immunother. 14, 2618-2623 (2018).

21. Yanagawa, S. et al. Development of a humanized mouse model to analyze antibodies specific for human leukocyte antigen (HLA). PLoS One 16, 1-20 (2021).

22. Lachmann, N. et al. Anti-human leukocyte antigen and donor-specific antibodies detected by luminex posttransplant serve as biomarkers for chronic rejection of renal allografts. Transplantation $\mathbf{8 7}$, 1505-1513 (2009).

23. O'Leary, J. G. et al. High mean fluorescence intensity donor-specific anti-HLA antibodies associated with chronic rejection postliver transplant. Am. J. Transplant. 11, 1868-1876 (2011).

\section{Tables}

Table 1. Characteristics of CDNA-uPA/SCID/Rag2 ${ }^{-/ /} / \mathrm{Jak3}^{-/-}$mice 


\begin{tabular}{|c|c|c|c|c|c|c|}
\hline uPA & Sex & $\mathrm{n}$ & $\begin{array}{l}\text { Survival rate until } \\
11 \text { weeks (\%) }\end{array}$ & $\begin{array}{l}\text { Body weight at } \\
11 \text { weeks }(\mathrm{g})\end{array}$ & $\begin{array}{l}\text { HSA level at } \\
11 \text { weeks } \\
\left(\times 10^{6} \mathrm{ng} / \mathrm{mL}\right)\end{array}$ & $\begin{array}{l}\text { HSA level at } \\
13 \text { weeks } \\
\left(\times 10^{6} \mathrm{ng} / \mathrm{mL}\right)\end{array}$ \\
\hline \multirow[t]{2}{*}{ Homo } & Male & 8 & 100 & $15.7 \pm 2.3(n=8)$ & $9.6 \pm 1.0$ & $\begin{array}{l}10.7 \pm 1.3 \\
(n=6)\end{array}$ \\
\hline & Female & 17 & 88.2 & $14.6 \pm 2.4(n=15)$ & $8.7 \pm 1.5$ & $\begin{array}{l}10.1 \pm 1.4 \\
(n=6)\end{array}$ \\
\hline \multirow[t]{2}{*}{ Hemi } & Male & 23 & 100 & $17.4 \pm 3.8(n=23)$ & $6.8 \pm 2.1$ & $\begin{array}{l}8.2 \pm 1.8 \\
(n=20)\end{array}$ \\
\hline & Female & 41 & 97.6 & $16.9 \pm 2.5(n=40)$ & $5.8 \pm 2.0$ & $\begin{array}{l}7.4 \pm 1.5 \\
(n=30)\end{array}$ \\
\hline
\end{tabular}

uPA, urokinase-type plasminogen activator; HSA, human serum albumin

Table 2. Characteristics of peripheral bood mononuclear cells and hepatocytes

\begin{tabular}{lllllll} 
Case & Background & HBsAg & HBV DNA & HLA-A & HLA-B & HLA-DR \\
\hline Case 1 & Healthy volunteer & Negative & Undetectable & $24: 33$ & $44: 52$ & $13: 15$ \\
\hline Case 2 & Acute hepatitis B & Negative & Undetectable & $24: 24$ & $52: 52$ & $15: 15$ \\
\hline Case 3 & Chronic hepatitis B & Positive & Undetectable & $24: 24$ & $52: 54$ & $4: 15$ \\
\hline BD195 & Human hepatocyte & N.A. & N.A. & $24: 24$ & $52: 61$ & $8: 14$
\end{tabular}

HBsAg, hepatitis B surface antigen; HLA, human leukocyte antigen; N.A., not applicable

Table3. Anti-HLA antibody appearing after PBMC administration in human hepatocyte chimeric cDNAUPA/SCID/Rag2 ${ }^{-/} / \mathrm{Jak}^{-/-}$mice. 


\begin{tabular}{llll} 
Donor & HBV infection & HLA-A & HLA-B \\
\hline- & - & N.D. & N.D. \\
\hline Donor 1 & - & A11.1, A31, A30, A26 & B61 \\
\hline Donor 1 & - & N.D. & B54, B27, B62 \\
\hline Donor 1 & + & N.D. & N.D. \\
\hline Donor 1 & + & N.D. & N.D. \\
\hline Donor 2 & + & N.D. & B7 \\
\hline Donor 2 & + & N.D. & B61, B67 \\
\hline Donor 2 & + & A31 & B7, B61 \\
\hline Donor 2 & + & N.D. & B37 \\
\hline Donor 3 & + & N.D. & N.D.
\end{tabular}

B61, Anti-HLA antibody common7 to transplanted hepatocytes; N.D., not detected.

Figures 
Figure 1

A
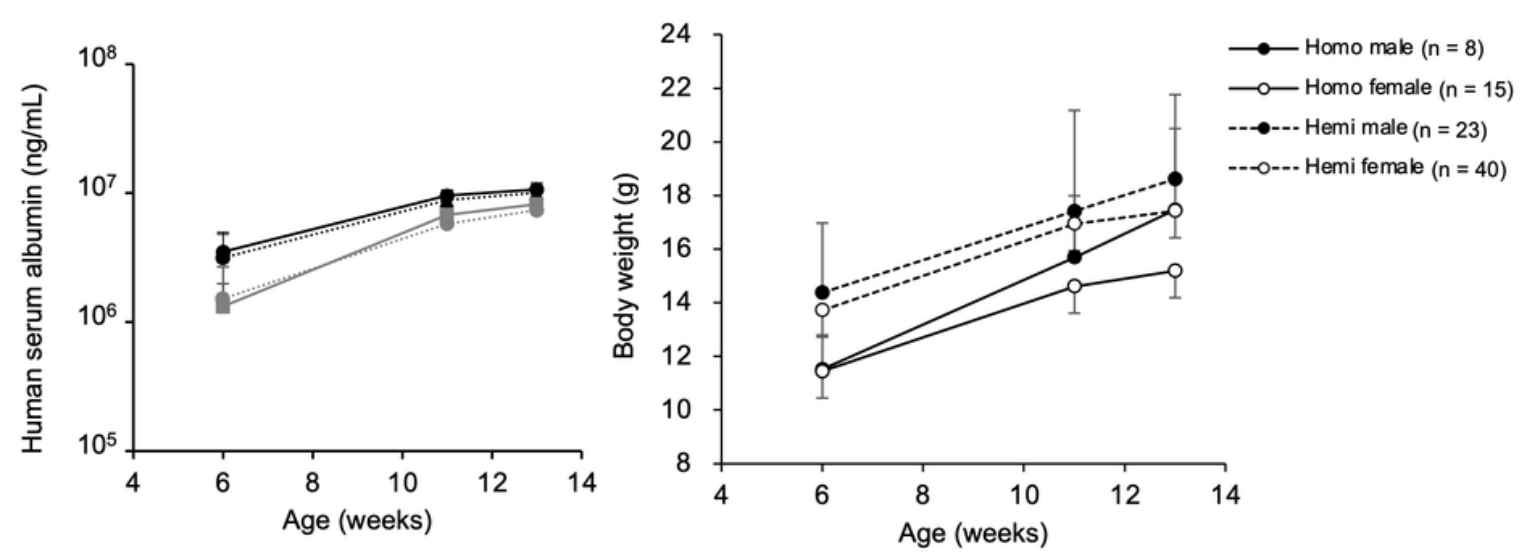

B

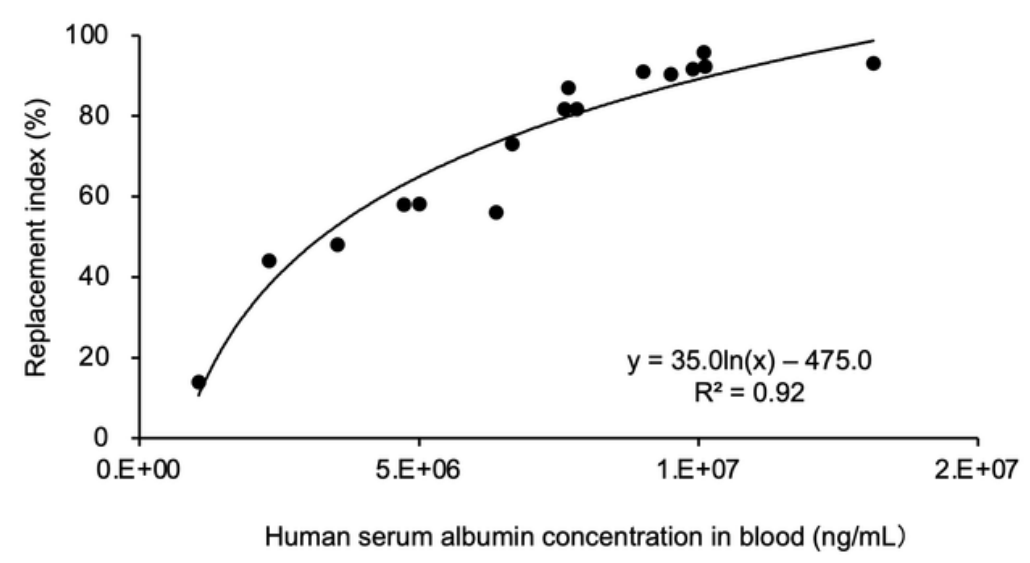

Figure 1

Human serum albumin concentrations and body weight in human hepatocyte-replaced cDNAuPA/SCID/Rag2-/-/Jak3-/- mice. cDNA-uPA/SCID/Rag2-/-/Jak3-/- mice were transplatend with human hepatocytes. (A) Changes in human serum albumin concnetrations and body weight in mice. (B) Correlation between replacement index and human serum albumin concentrations at 17-week-old. 


\section{Figure 2}

A

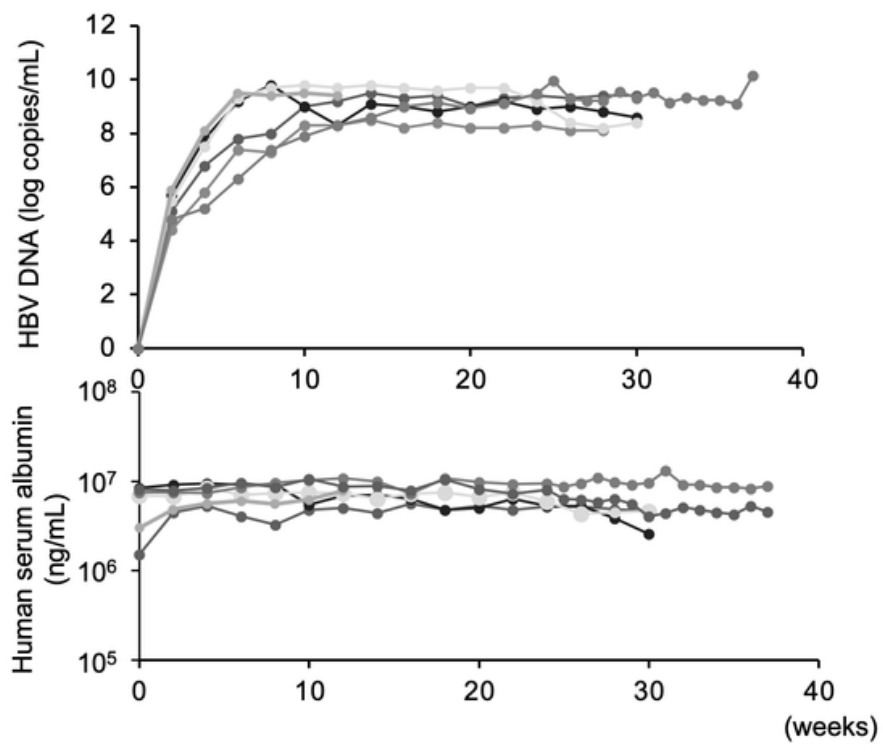

B

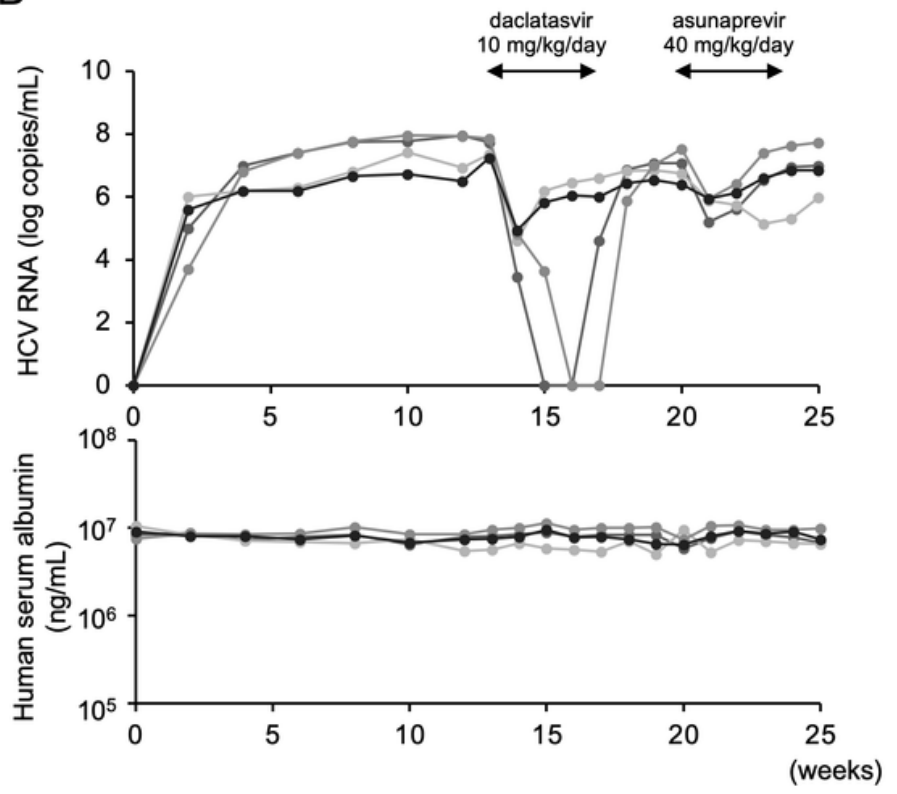

Figure 2

Hepatitis virus infection in human hepatocyte-replaced cDNA-uPA/SCID/Rag2-/-/Jak3-/- mice. Ten human hepatocyte-transplantd cDNA-UPA/SCID/Rag2-/-/Jak3-/- mice were infected with either HBV (A) or HCV (B). HCV-infectd mice were treated with daclatasvir for four weeks and then treated with asunaprevir for four weeks. Changes in serum HBV DNA or HCV RNA titer and human serum albumin concentration in each mouse are shown. 


\section{Figure 3}

A

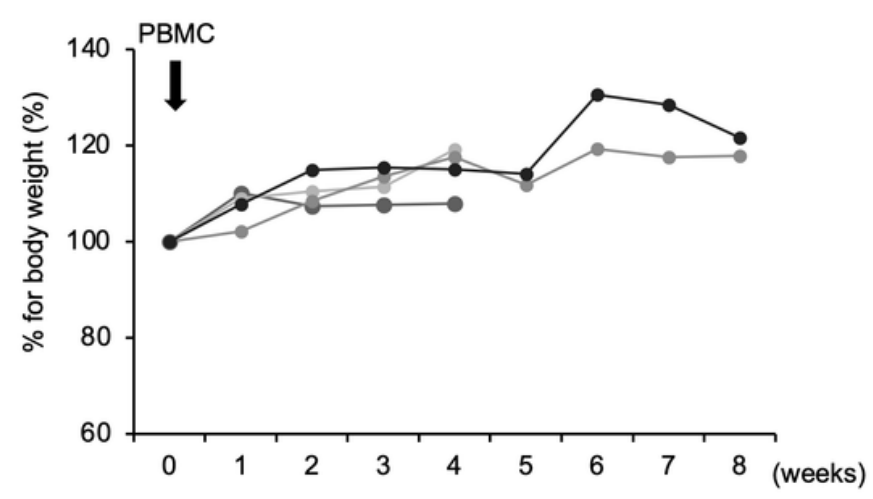

B

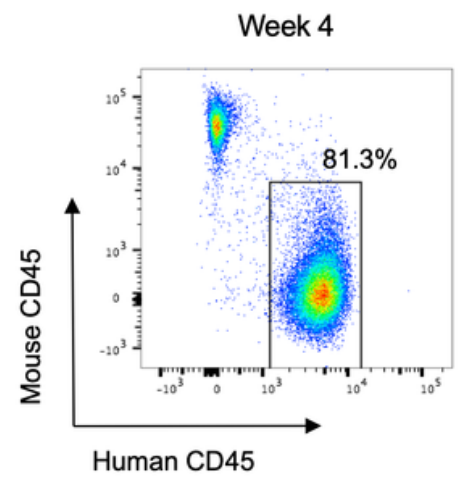

Week 8

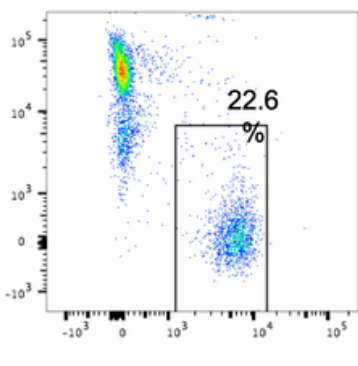

\section{Figure 3}

PBMC transplantation into CDNA-uPA/SCID/Rag2-/-/Jak3-/- mice. Each of four cDNAuPA/SCID/Rag2-/-/Jak3-/-/- mice without transplanted human hepatocytes was administered human peripheral blood mononuclear cells (PBMCs) obtained from a healthy volunteer. (A) Changes in the percent of body weight in each mouse. (B) Proportions of human mononuclear cells in mouse liver infiltrating cells in two mice, at four and eight weeks after PBMCs transplantation, respectively. 


\section{Figure 4}
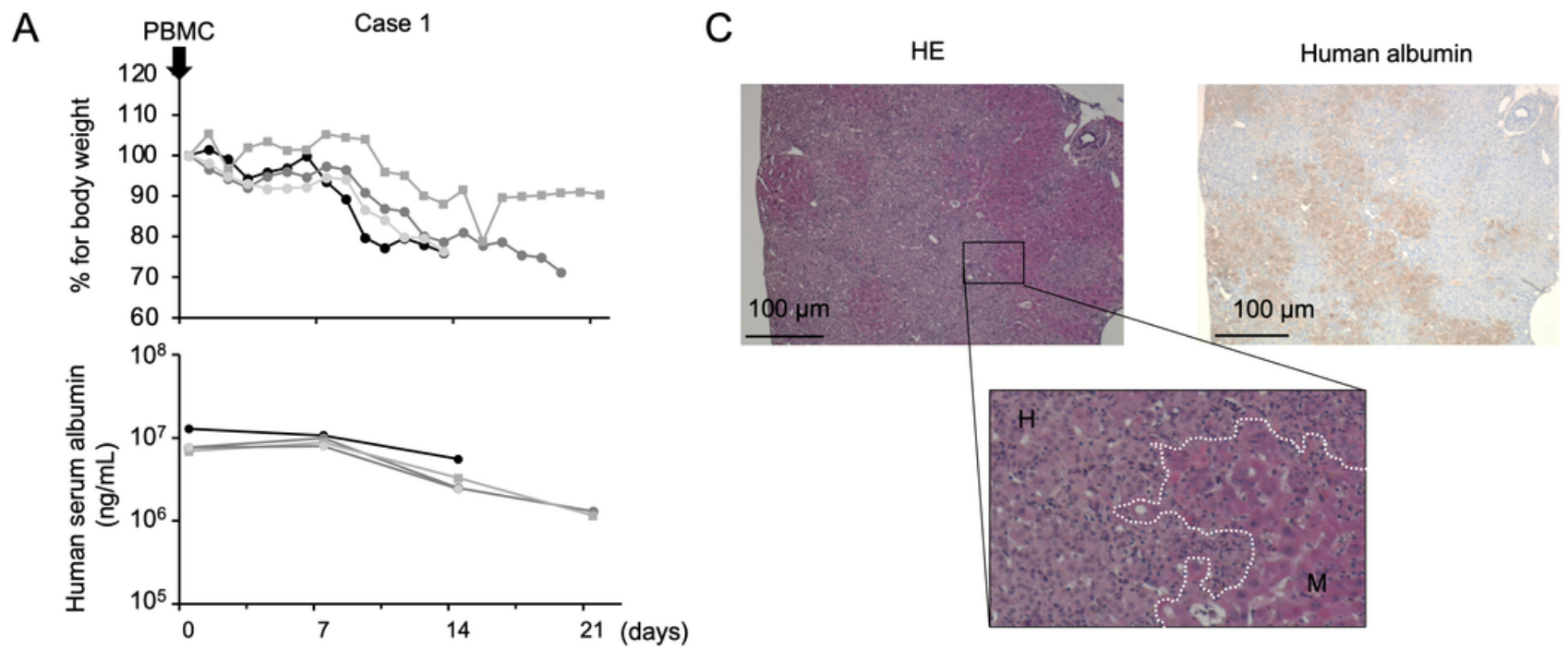

B
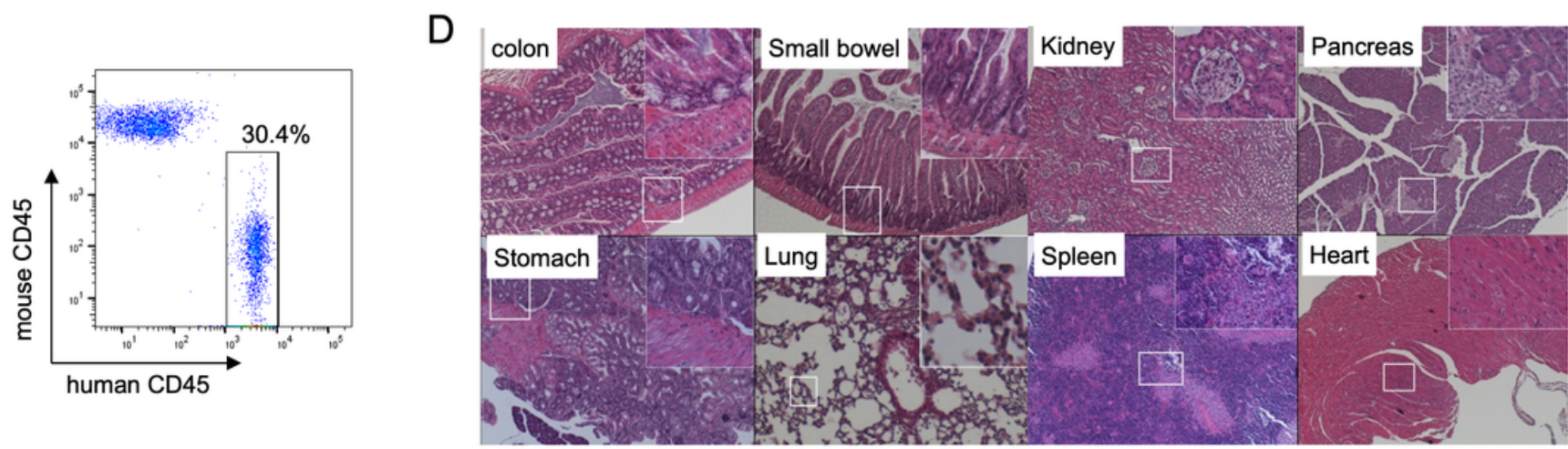

\section{Figure 4}

PBMC administration into human hepatocyte-transplanted cDNA-uPA/SCID/Rag2-/-/Jak3-/- mice. Each of four human hepatocyte-transplanted CDNA-uPA/SCID/Rag2-/-/Jak3-/- mice was administered human peripheral blood mononuclear cells (PBMCs) obtained from a healthy volunteer. (A) Changes in percent of body weight and human serum albumin concentration in each mouse. (B) Proportions of human mononuclear cells in mouse liver infiltrating cells. (C) Histological analysis of mouse liver samples. Liver samples at two weeks after injection of human PBMCs were stained with hematoxylin-eosin (HE) or antihuman albumin antibody. Regions are shown as human $(H)$ and mouse $(M)$ hepatocytes, respectively (original magnification, $\times 40$ or $\times 200$ ). (D) HE staining of colon, small intestine, kidney, pancreas, stomach, lung, spleen and heart (original magnification, $\times 40$ ). 
Figure 5

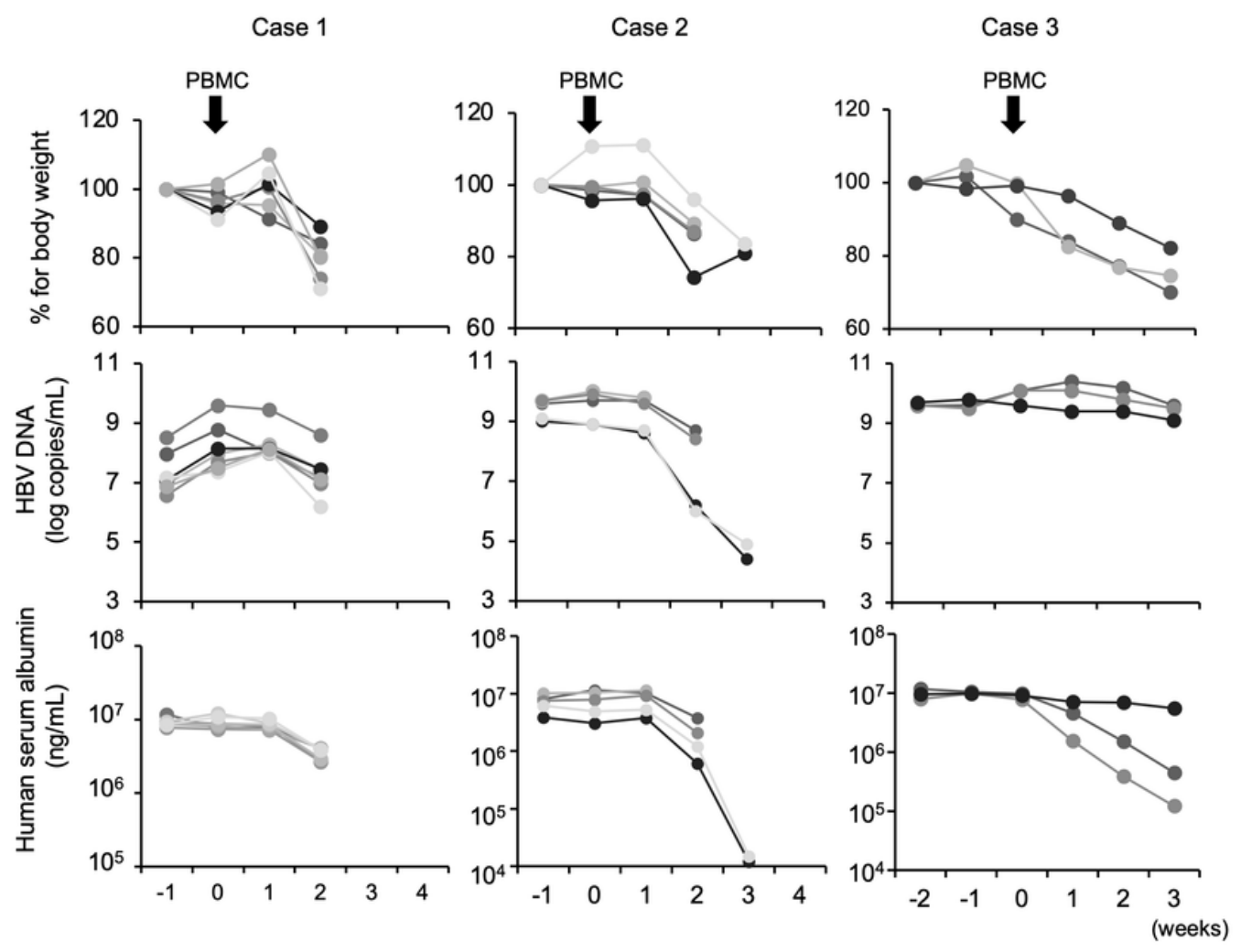

Figure 5

PBMC administration into HBV-infected cDNA-uPA/SCID/Rag2-/-/Jak3-/- mice. Human hepatocytetransplanted cDNA-uPA/SCID/Rag2-/-/Jak3-/- mice were infected with HBV and administered human peripheral blood mononuclear cells (PBMCs) obtained from a healthy volunteer (Case 1), a patient recovered from an episode of acute hepatitis B (Case 2) or a chronic HBV-infected patient (Case 3). Changes in body weight, serum HBV DNA level and human serum albumin concentration in each mouse are shown. 
Figure 6

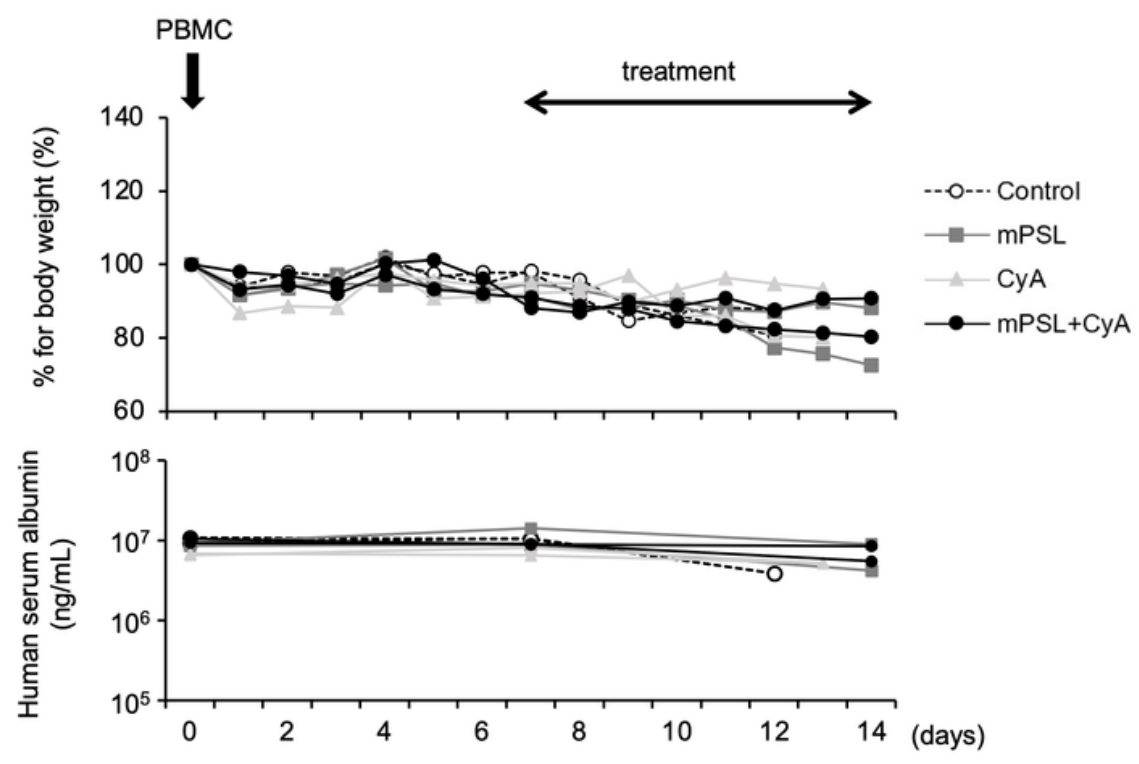

Figure 6

Treatment with immunosupression for human PBMC-tranplanted mice. Human hepatocyte-transplantd CDNA-UPA/SCID/Rag2-/-/Jak3-/- mice were administered human peripheral blood mononuclear cells (PBMCs) obtained from Case 1. One week after human PBMC transplantation, each of eight mice was treated with or without (Control) methylprednisolone ( $\mathrm{mPSL}$ ), cyclosporine A (CyA), or combination of mPSL and CyA for seven days. Changes in the percent of body weight and human serum albumin concentration in each mouse are shown. 